\title{
Short- and long-lasting behavioral and neurochemical adaptations: relationship with patterns of cocaine administration and expectation of drug effects in rats
}

\author{
S Puig ${ }^{1,2,3}, F_{\text {Noble }}^{1,2,3}$ and N Benturquia ${ }^{1,2,3}$
}

Cocaine dependence is a significant public health problem, characterized by periods of abstinence. Chronic exposure to drugs of abuse induces important modifications on neuronal systems, including the dopaminergic system. The pattern of administration is an important factor that should be taken into consideration to study the neuroadaptations. We compared the effects of intermittent (once daily) and binge (three times a day) cocaine treatments for 1 (WD1) and 14 (WD14) days after the last cocaine injection on spontaneous locomotor activity and dopamine (DA) levels in the nucleus accumbens (Nac). The intermittent treatment led to a spontaneous increase in DA (WD1/WD14), and in locomotor activity (WD1) at the exact hour which rats were habituated to receive a cocaine injection. These results underline that taking into consideration the hours of the day at which the experiments are performed is crucial. We also investigated these behavioral and neurochemical adaptations in response to an acute cocaine challenge on WD1 and WD14. We observed that only the binge treatment led to sensitization of locomotor effects of cocaine, associated to a DA release sensitization in the Nac, whereas the intermittent treatment did not. We demonstrate that two different patterns of administration induced distinct behavioral and neurochemical consequences. We unambiguously demonstrated that the intermittent treatment induced drug expectation associated with higher basal DA level in the Nac when measured at the time of chronic cocaine injection and that the binge treatment led to behavioral and sensitization effects of cocaine.

Translational Psychiatry (2012) 2, e175; doi:10.1038/tp.2012.103; published online 23 October 2012

\section{Introduction}

Cocaine is a widely abused drug that possesses a significant health concern with major social and economic ramifications. Cocaine addiction is a process that generally starts with recreational use and deteriorates over time into a compulsive and chronic-relapsing drug taking disorder. ${ }^{1}$ Despite longstanding efforts to identify compounds capable of selectively inhibiting the addictive effects of cocaine, there are currently no approved medications for the treatment of cocaine abuse or toxicity. Nevertheless, addiction is a complex pathology, multifactorial, which may explain why the clinical trials performed to evaluate the therapeutic potential of pharmacotherapies are unsatisfactory. Several parameters can and should be taken into considerations, including the pattern of administration, and the transient or long-lasting neurochemical and behavioral abnormalities following drug administration.

To achieve a good understanding of the neuroadaptations induced by cocaine, the use of animal models provides enormous potential, through a coordinated analysis of brain function and behavior. As previously mentioned, the pattern of administration and the kinetics of the neuroadaptations are important factors that should be taken into consideration. Several studies reinforce this hypothesis. ${ }^{2-5}$ In addition, our laboratory has already studied the effects of manipulating the frequency of available injections by using a discrete trials procedure, showing that two different patterns of morphine treatment induced distinct behavioral and neurochemical consequences with different time courses. ${ }^{6}$ Cocaine exerts its pharmacological action through the monoamine systems. In particular, dopamine (DA) projections to the striatum have been implicated greatly in reinforcement and motor behaviors produced by cocaine. ${ }^{7}$ Thus, the first aim of our study was to investigate and compare the spontaneous behavioral and neurochemical consequences of two 14-day chronic cocaine pretreatment regimens (intermittent versus binge), both of which are used extensively in laboratories to mimic patterns of cocaine abuse in humans. Extracellular DA levels were evaluated 1 (WD1) and 14 (WD14) days after the last cocaine injection. In parallel to this, the locomotor activity of the animals was measured during $24 \mathrm{~h}$ on WD1 and WD14. These experiments were performed either exactly at the hours at which rats were habituated to receive a cocaine injection or at another hour of the day. We showed that intermittent cocaine (IC) and binge cocaine (BC) treatments induced distinct behavioral and neurochemical consequences with different time courses. The second aim of this study was to investigate the behavioral and neurochemical responses to an acute cocaine challenge following chronic cocaine pretreatment.

\footnotetext{
${ }^{1}$ Centre National de la Recherche Scientifique, Unité Mixte de Recherche 8206, Paris, France; ${ }^{2}$ Institut national de la Santé et de la Recherche Médicale, U 705, Paris, France and ${ }^{3}$ Université Paris Descartes, Laboratoire de Neuropsychopharmacologie des Addictions, CNRS UMR 8206-INSERM U 705, Faculté de Pharmacie, Paris, France

Correspondence: Dr F Noble, Université Paris Descartes, Laboratoire de Neuropsychopharmacologie des Addictions, CNRS UMR 8206-INSERM U 705, Faculté de Pharmacie, 4, avenue de l'Observatoire, 75006 Paris, France.

E-mail: florence.noble@ parisdescartes.fr

Keywords: cocaine; dopamine; expectation; locomotor sensitization; pattern of administration

Received 5 June 2012; revised 17 July 2012; accepted 18 August 2012
} 
Here again, we showed that the patterns of chronic cocaine administration induced distinct consequences, with behavioral and neurochemical sensitization only observed following the binge treatment.

\section{Materials and methods}

Animals. Male Sprague-Dawley rats (Janvier, Le GenestSaint-Isle, France) weighing 275-300 g at the beginning of the treatments were housed on a 12-h light/dark cycle in a temperature- $\left(22 \pm 1^{\circ} \mathrm{C}\right)$ and humidity-controlled $(50 \pm 5 \%)$ environment and had access to food and water ad libitum. Animals were treated in accordance with the NIH Guidelines for the Care and Use of Laboratory Animals ${ }^{8}$ (1996) and in agreement with the local ethical committee. The number of animals used and their suffering was minimized in all experiments designed.

Cocaine treatments. Animals were treated by an IC or a BC administration profile, consisting in intraperitoneal (i.p.) injections of $20 \mathrm{mg} \mathrm{kg}^{-1}$ cocaine hydrochloride (Francopia, Anthony, France), dissolved in saline $(0.9 \%(\mathrm{w} / \mathrm{v}) \mathrm{NaCl})$ during 14 days, once daily at 1000 hours for the intermittent profile and three times a day at 1000, 1300 and 1600 hours for the binge profile. All animals received $1 \mathrm{ml} \mathrm{kg}^{-1}$ of their bodyweight. Control groups were treated with saline in the same conditions (intermittent saline (IS) or binge saline (BS)). Immediately after injection, rats were returned to their home cages.

Behavioral study. Locomotor activity was evaluated on WD1 and WD14 after the end of the treatments (see Supplementary Figure 1), in an actimeter (Immetronic, Pessac, France) composed of eight cages $(34 \times 21 \times 19 \mathrm{~cm})$ under low illumination ( $<5$ lux). One rat was placed in each box to record its movements. Displacements were measured by photocell beams located across the long axis and above the floor. Vertical and horizontal activity was recorded and expressed in scores (mean \pm s.e.m.) as the total number of interruption of the photocell beams. The $12 / 12 \mathrm{~h}$ light/dark cycle was respected.

For the spontaneous locomotor activity on WD1: animals were placed during $24 \mathrm{~h}$ in the actimeter $2 \mathrm{~h}$ after the last injection of the chronic treatment; and on WD14: the same protocol was used, animals were placed during $24 \mathrm{~h}$ in the actimeter in day 27 plus $2 \mathrm{~h}$.

In other groups of animals, we have evaluated the locomotor activity response to cocaine $\left(20 \mathrm{mg} \mathrm{kg}^{-1}\right.$, i.p.) or saline challenge; animals were challenged out of the usual hours of the injection treatment (on WD1 and WD14) and immediately placed in the actimeter during $1 \mathrm{~h}$.

\section{Neurochemical study}

Surgery: Rats were anesthetized by an i.p. injection of a mixture of ketamine/xylazine $\left(80 / 10 \mathrm{mg} \mathrm{kg}^{-1}\right)$ and placed in a stereotaxic apparatus (Unimécanique, Eaubonne, France). A guide cannula (CMA 12, Phymep, Paris, France) was stereotaxically implanted in the nucleus accumbens core (Nacc). The coordinates, taken from the atlas of Paxinos and Watson (1998), ${ }^{9}$ were $+1.6 \mathrm{~mm}$ anterior to the interaural, $+1.4 \mathrm{~mm}$ lateral to the midline and $-6.0 \mathrm{~mm}$ under the skull surface. Animals were used for experiments after a recovery period of 1 week.

Judgments about cannula placements in the Nacc were made by an observer who was blind to the results obtained with individual rats. Only the rats implanted into the Nacc were analyzed in the study (the fail rate for the implantation was 5/ 100, Supplementary Figure 2).

Microdialysis procedures were done on WD1 and WD14 after the end of the treatments (Supplementary Figure 1b). Rats were gently restrained, the stylus was removed from the guide cannula and the probe (CMA 12 Ellit, 2 mm, Phymep) was implanted and perfused at $2 \mu \mathrm{min}^{-1}$. The perfusate consisted in artificial cerebrospinal fluid containing (in $\mathrm{mM}$ ) $\mathrm{NaCl} 140, \mathrm{KCl} 4, \mathrm{MgCl}_{2} 1, \mathrm{NaH}_{2} \mathrm{PO}_{4} 0.1, \mathrm{Na}_{2} \mathrm{HPO}_{4} 1.9$ and $\mathrm{CaCl}_{2} 1.2(\mathrm{pH}=7.4)$. After $2 \mathrm{~h}$ equilibration of the microdialysis membrane, samples were collected every $30 \mathrm{~min}$ in tubes containing $5 \mu \mathrm{HClO}_{4}$ of $0.4 \mathrm{M}$ to prevent DA oxidation and conserved at $-80^{\circ} \mathrm{C}$ until the quantification.

For the measure of the DA level at injection hours and evaluation of the neurochemical response to a cocaine challenge, the samples collection occurred on WD1 and WD14.

For the DA basal level, two samples was collected out of the usual hours of chronic injection treatment, then two other samples was collected at the exact hour when rats were habituated to receive cocaine without any injection. We have evaluated DA release to a cocaine challenge; few hours later, out of the usual hours of injection chronic treatment, two samples were collected following i.p. challenge injection (cocaine or saline).

To exclude effect of association drug-spatial cues, drug treatment was performed in a different room of the microdialysis room.

$D A$ analysis: DA content was determined as previously described $^{10}$ using HPLC apparatus coupled to electrochemical detector (Coulochem III, ESA, Chelmsford, MA, USA).

Statistical analysis. The neurochemical studies in drugfree conditions were analyzed by a two-tailed t-test. A oneway analysis of variance (one-way ANOVA) was used to analyze locomotor activity in response to a cocaine challenge. A two-way ANOVA was used for the analysis of the locomotor activity measured during $24 \mathrm{~h}$ in drug-free conditions (treatment $\times$ time), and for the analysis of DA levels in the Nacc following a cocaine challenge injection (treatment $x$ microdialysate). The Bonferroni test was used for post-hoc comparisons.

\section{Results}

Spontaneous neurochemical and behavioral consequences of chronic cocaine treatment

Spontaneous locomotor activity. The measure during $24 \mathrm{~h}$ of locomotor activity $2 \mathrm{~h}$ after the last injection of treatment showed differences in behavioral regulation (Figure 1). A twoway ANOVA showed a significant treatment $\times$ time interaction for the intermittent profile on WD1 $\left(F_{(1,20)}=2.36, P<0.001\right)$ (Figure 1a), with a significant time effect $\left(F_{(1,20)}=5.39\right.$, 
Protocol paradigm
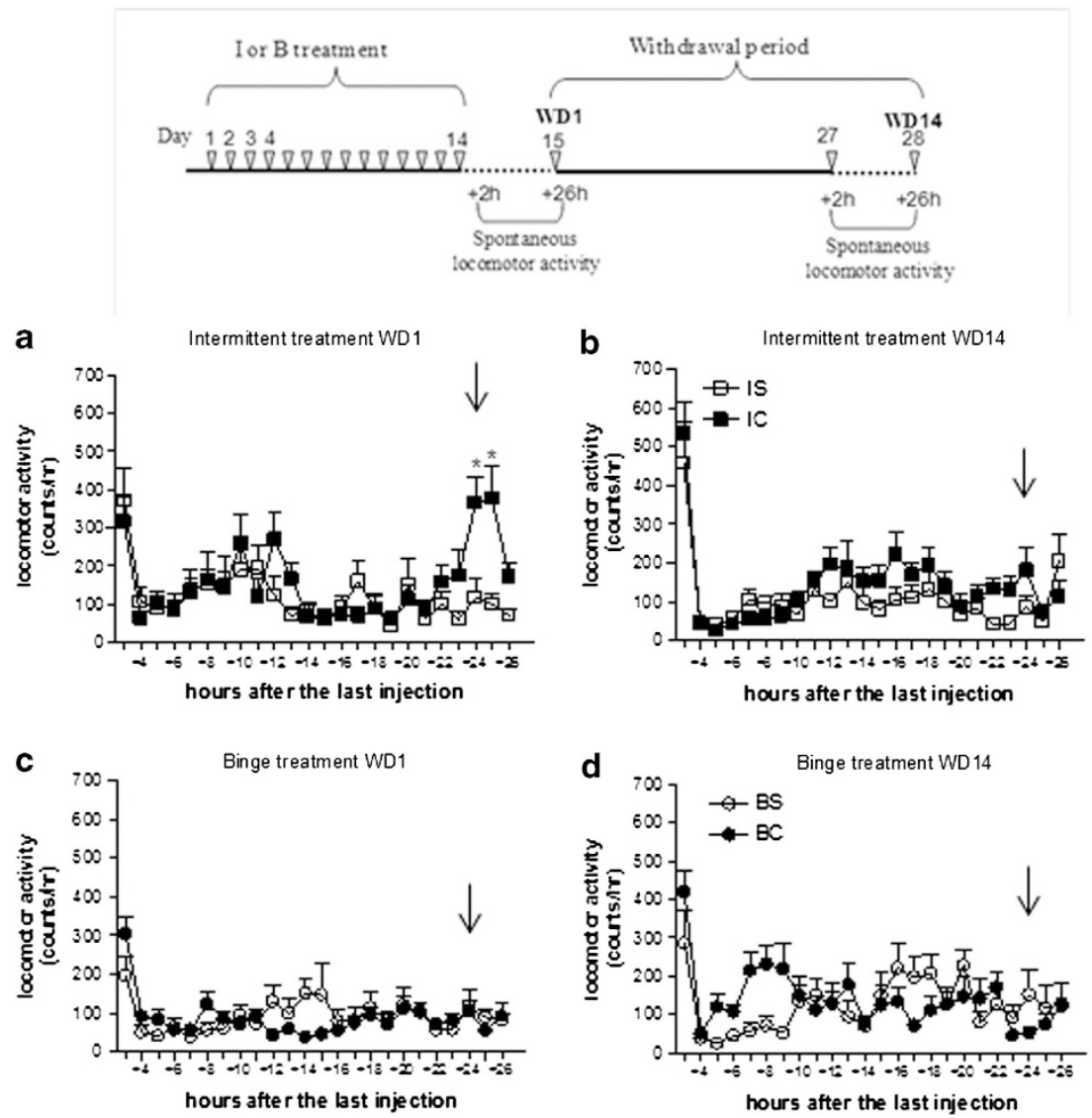

Figure 1 Spontaneous locomotor activity. On WD1 (a and c) and on WD14 (b and d), locomotor activity was measured during $24 \mathrm{~h}$ according to the protocol paradigm. The arrows indicate the usual hours of injection during the intermittent or binge treatment. Results were expressed in counts per hour (mean \pm s.e.m.). ${ }^{*} P<0.05$, Bonferroni test $(n=9-14)$. B, binge; BC, binge cocaine; BS, binge saline; I, intermittent; IC, intermittent cocaine; IS, intermittent saline.

$P<0.0001)$, but no treatment effect $\left(\mathrm{F}_{(1,20)}=1.39\right.$, NS). Posthoc analysis showed an increase of locomotion for the IC group as compared with the IS group during the 24th and 25th hour after the last injection, corresponding exactly to the hour when rats were habituated to be injected. This effect did not persist after 14 days of withdrawal (Figure $1 \mathrm{~b}$ ) as no significant treatment $\times$ time interaction $\left(F_{(1,19)}=1.05\right.$, NS) was observed, with no treatment effect $\left(F_{(1,19)}=1.75\right.$, NS), but a significant time effect $\left(F_{(1,19)}=12.05, P<0.0001\right)$.

Unlike intermittent-treated animals, the locomotion of binge animals on WD1 or on WD14 was not changed during the $24 \mathrm{~h}$ of measurement in comparison with the saline (Figures 1c and d). However, significant treatment $\times$ time interactions were observed, which were mainly due to a higher locomotor activity measured during the first hour, in the cocainetreated animals in comparison with saline group (WD1 Figure 1c: treatment $\times$ time interaction: $F_{(1,25)}=2.15$, $P<0.01$, treatment effect: $F_{(1,25)}=0.14$, NS and time effect: $\mathrm{F}_{(1,25)}=4.31, P<0.0001$; WD14 Figure 1d: treatment $\times$ time interaction: $F_{(1,21)}=2.26, \quad P<0.001$, treatment effect: $\mathrm{F}_{(1,21)}=0.36$, NS, and time effect: $\left.\mathrm{F}_{(1,21)}=4.21, P<0.0001\right)$.

As shown in Figure 1d, we observed an increase in locomotor activity at day 27 after the beginning of the treatment in the $\mathrm{BC}$ group as compared with the saline group between time point +4 and $+10 \mathrm{~h}$. However, this increase was not statistically significant.

Basal extracellular level of DA. At a time that did not match the hours of injection, the levels of extracellular DA was evaluated in the Nacc using microdialysis in awake and freely moving rats.

As shown in Figure 2a, two tailed $t$-test revealed a decrease of basal DA levels, 1 day after the end of the treatment, for the $\mathrm{IC}$ and $\mathrm{BC}$ groups in comparison with their respective saline control group (Figure 2a, IC: $n=15, \mathrm{t}_{(13)}=2.49, P<0.05$; $\mathrm{BC}$ $\left.n=13, \mathrm{t}_{(11)}=2.50, P<0.05\right)$. This effect persisted after 14 days of withdrawal for the IC group (WD14, Figure $2 b, n=15$, $\left.\mathrm{t}_{(13)}=2.57, P<0.05\right)$. No significant difference in basal levels of DA for the BC group in comparison with the BS group was observed.

Injection hour extracellular level of DA. As shown in Figure 3, two tailed $t$-test revealed a significant increase of extracellular DA levels in the Nacc core, 1 day after the end of treatments as compared to the basal levels, at the time corresponding to the injection hours for both treatment profiles, IC $\left(n=20, \mathrm{t}_{(18)}=3.81, P<0.01\right)$ and $\mathrm{BC}$ 

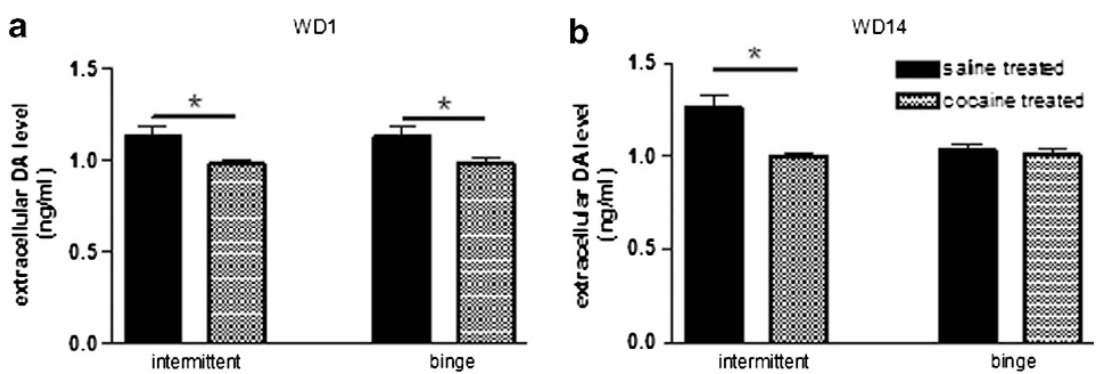

Figure 2 Basal extracellular level of dopamine (DA) in the nucleus accumbens core (Nacc). Two dialysis samples were collected every 30 min on WD1 (a) and on WD14 (b) to determine basal level of DA in the Nacc out of the usual hours of injection treatment. Each column represents the extracellular dopamine levels in ng $\mathrm{ml}^{-1}$ (mean \pm s.e.m.). ${ }^{*} P<0.05$ two-tailed $t$-test $(n=6-10)$.
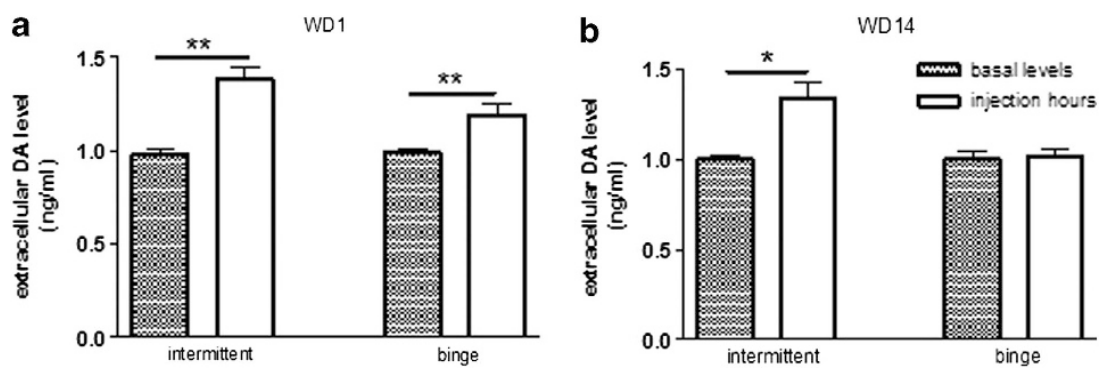

Figure 3 Injection hour extracellular level of dopamine (DA) in the nucleus accumbens core (Nacc). Two dialysis samples were collected every 30 min on WD1 (a) and on WD14 (b) to determine the basal level of DA in the Nacc, then two dialysis samples were collected exactly at the time of the usual hours of injection for each treatment without a new injection. Each column represents the extracellular dopamine levels in $\mathrm{ng} \mathrm{ml}^{-1}$ (mean \pm s.e.m.). ${ }^{*} P<0.05,{ }^{* *} P<0.01$, two-tailed $t$-test $(n=6-14)$.

$\left(n=12, \mathrm{t}_{(10)}=2.36, P<0.01\right)$. Interestingly, this effect was still observed on WD14 only in the IC group $(n=15$, $\mathrm{t}\left({ }_{13)}=2.86, P<0.05\right)$ (Figure $\left.3 \mathrm{~b}\right)$.

Behavioral and neurochemical responses to a cocaine challenge (out of the usual hours of injection treatment) Locomotor activity. As shown in Figure 4, the locomotor activity responses observed for $1 \mathrm{~h}$ immediately following saline or cocaine administration on WD1 or WD14 days were not modified in IC group in comparison with the control group. One-way ANOVA showed a significant effect of cocaine challenge, with an increase in the locomotor activity in both cocaine- and saline-treated animals (WD1, Figure 4a: intermittent: $F_{(3,43)}=20.67, P<0.0001$; WD14, Figure 4b, $\left.F_{(3,44)}=23.50, P<0.0001\right)$.

For the binge treatment, Figure $4 \mathrm{c}$ clearly showed a significant sensitization to the hyperlocomotor effect induced by cocaine on WD1 $\left(F_{(3,41)}=40.89, P<0.0001\right.$; Bonferroni BS challenge cocaine vs BC challenge cocaine $P<0.01$ ). On WD14, the locomotor activity was not modified in comparison with the control group. One-way ANOVA showed a significant effect of cocaine challenge, with an increase in the locomotor activity in both cocaine- and saline-treated animals $\left(F_{(3,40)}=20.21, P<0.0001\right)$. No differences in the locomotor activity responses were observed after a cocaine challenge, between the cocaine- and saline-treated animals.

Extracellular DA level. As shown in Figure $5 a$, cocaine challenge on WD1 induced an increase in the extracellular DA levels in IC and IS-treated rats as compared with animals that received a saline challenge treatment (interaction: $F_{(3,21)}=3.03, P<0.01$, treatment effect: $F_{(3,21)}=4.72$, $P<0.05$, time effect: $\left.F_{(3,21)}=11.76, P<0.0001,\right)$. Similar results were observed on WD14 (two-way ANOVA: interaction: $F_{(3,20)}=2.84, P<0.01$, treatment effect: $F_{(3,20)}=3.39$, $P<0.05$, time effect: $F_{(3,20)}=8.76, P<0.0001$ ) (Figure $5 b$ ).

In the BC profile on WD1, the two-way ANOVA showed a significant interaction $\left(F_{(3,24)}=2.37, P<0.05\right)$, treatment effect $\left(\mathrm{F}_{(3,24)}=3.66, P<0.05\right)$ and time effect $\left(\mathrm{F}_{(3,24)}=8.76\right.$, $P<0.0001$ ). Post-hoc analysis revealed (Figure $5 \mathrm{C}$ ) a greater increase of extracellular DA level following cocaine challenge in $\mathrm{BC}$ group $(+164 \% \pm 53.83$ of basal levels), in comparison with the control group that received cocaine for the first time $(+72 \% \pm 46.87$ of basal levels). As shown in Figure $5 \mathrm{~d}$, this neurochemical sensitization was not observed on WD14. (Two-way ANOVA: interaction: $\left.F_{(3,} 24\right)=2.29, P<0.05$, treatment effect: $F_{(3,24)}=5.39, P<0.01$, time effect: $F_{(3,24)}=$ 8.28, $P<0.001)$.

\section{Discussion}

In the present study, the short- and long-term behavioral and neurochemical consequences of two distinct cocaine pretreatment regimens ('intermittent' versus 'binge') were compared. The main finding of this study is that IC and BC treatments induce different behavioral and neurochemical adaptations, which may be long-lasting.

The results indicated that chronic cocaine administration led to a significant lowering of the basal DA levels in the Nacc for both intermittent and binge treatments. Interestingly, 
Protocol paradigm :

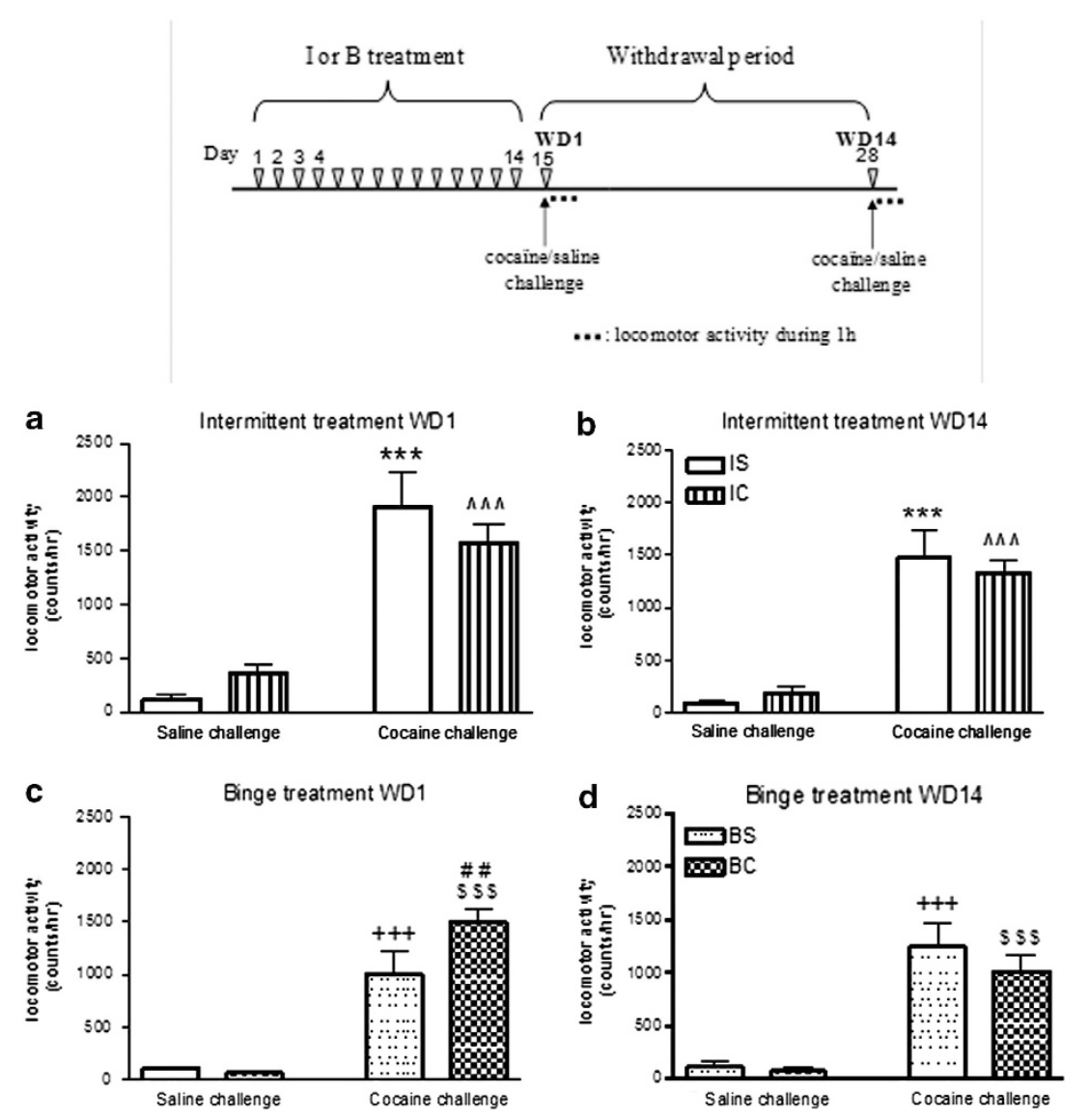

Figure 4 Locomotor response to an acute cocaine challenge. On WD1 (a and $\mathbf{c}$ ) and on WD14 (b and $\mathbf{d})$ animals received a cocaine or a saline acute challenge injection out of the usual hours of injection treatment. Locomotor activity was recorded during $1 \mathrm{~h}$ and expressed in counts per hour (mean \pm s.e.m.). ${ }^{* \star} P<0.0001$, IS saline challenge vs IS cocaine challenge. $\wedge \wedge \wedge P<0.001$ IC saline challenge vs IC cocaine challenge. ${ }^{+++} P<0.0001 \mathrm{BS}$ saline challenge vs BS cocaine challenge. ${ }^{\$ \$ \$} P<0.0001 \mathrm{BC}$ saline challenge vs $\mathrm{BC}$ cocaine challenge. ${ }^{\# \#} P<0.05$ BS cocaine challenge vs BC cocaine challenge; one-way ANOVA $(n=9-14)$. B, binge; BC, binge cocaine; BS, binge saline; I, intermittent; IC, intermittent cocaine; IS, intermittent saline.

this effect was long-lasting, as 14 days after the last injection a lower DA level was observed in IC group as compared with the IS group. However, it is interesting to note that the basal level for the BC treatment on WD14 seems be the same with the other cocaine groups. The decrease in DA level in the BS group observed on WD14 is complex to explain, although it is highly reproducible. BS rats received saline injection three times daily for 14 days, which may induce stress, well known to induce an inhibition of DA release in the Nacc. ${ }^{11,12}$

The decrease of DA levels observed in cocaine-treated groups as compared with saline groups are consistent with other studies showing a decrease in basal DA levels in Nacc following a chronic 'binge' cocaine injection paradigm, ${ }^{13}$ in rats injected with cocaine twice daily for 9 or 14 days, ${ }^{14,15}$ in rats that received a single daily injection of cocaine $10^{16}$ or 18 days ${ }^{17}$ and also in two mouse strains that received cocaine injection three times a day for 14 days. ${ }^{18}$ However, it is also important to note that some studies have shown no change, ${ }^{19,20}$ whereas others have shown an increase in the Nacc DA levels in rats that received a single daily injection of cocaine 10 days on WD1, WD3 and WD7 $7^{2}$ or on WD2 but not on WD12 and WD22. ${ }^{21}$ In the majority of these studies, cocaine was administered at doses ranging from 10 to $30 \mathrm{mg} \mathrm{kg}^{-1}$, and in most of the cases, extracellular DA level was measured $24 \mathrm{~h}$ after the last injection of cocaine, but with few indications regarding the specific hour of the day at which the microdialysis experiments were performed. However, our results demonstrate for the first time that this parameter is crucial and may explain the differences of results of the previous studies. Thus, when microdialysis experiments were performed at the exact hours, which rats were habituated to receive a cocaine injection, an increase in DA levels was observed. This effect was long-lasting in the intermittent group, as this regulation was still observed 14 days after the last injection of cocaine. Therefore, although a low extracellular DA level was observed in the Nacc following BC and IC treatments, an increase in the phasic release was observed at the usual hours of cocaine injection. Several hypotheses may be found in the literature regarding these regulations. For instance, the lower DA levels are correlated to an increase in the density of the DAT binding sites and to a supersensitivity of D2 autoreceptors in the DA terminals. ${ }^{22}$ On the other hand, several lines of evidence suggest that phasic release of DA 

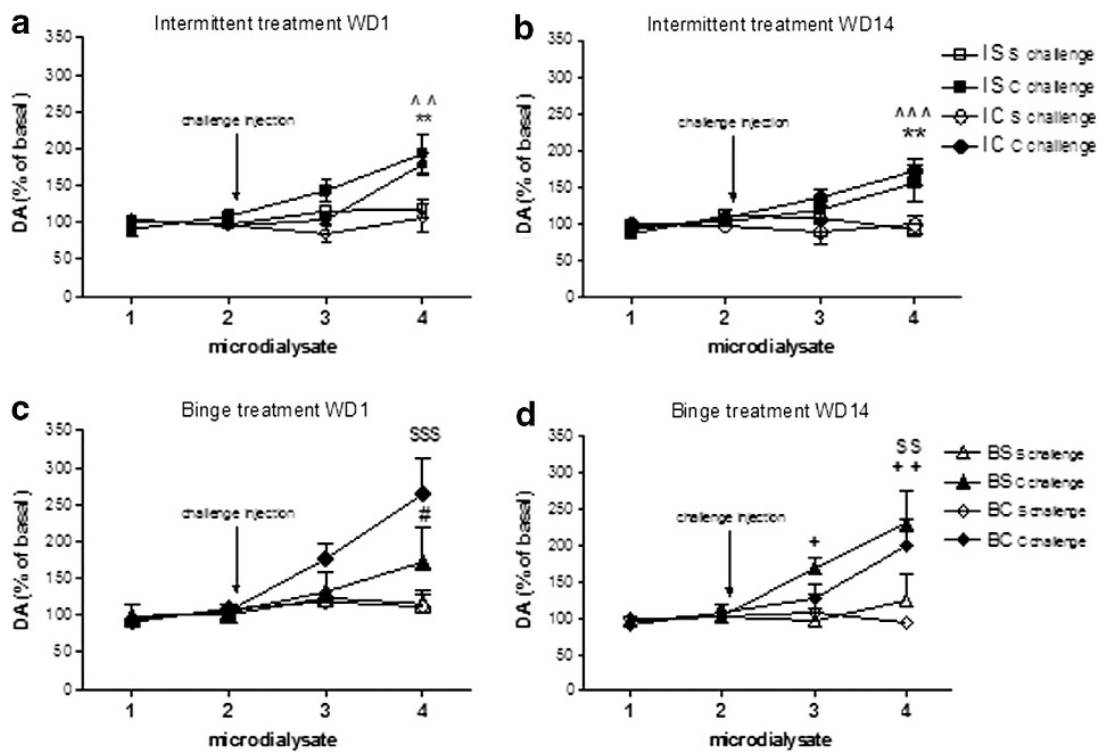

Figure 5 Extracellular dopamine (DA) in the nucleus accumbens core (Nacc) following an acute cocaine challenge. The dialysis samples were collected every 30 min out of the injection hour. The basal concentrations of DA in the Nac core, measured before the challenge injection, were (ng ml ${ }^{-1}$ ) for WD1, IS: $1.135 \pm 0.144, n=9$; IC: $0.979 \pm 0.060 n=14 ; \mathrm{BS}: 1.129 \pm 0.138, n=11 ; \mathrm{BC}: 0.987 \pm 0.069, n=11$ and for WD14, IS: $1.259 \pm 0.217, n=10 ;$ IC: $1.001 \pm 0.041, n=10$; BS: $1.032 \pm 0.076, n=12$; BC: $1.005 \pm 0.098, n=12$. Results were expressed as percent (mean \pm s.e.m.) of baseline value obtained before a challenge administration of cocaine or saline (black arrow) out of the usual hours of injection treatment. ${ }^{* \star} P<0.01$ IS saline challenge vs IS cocaine challenge. $M P<0.01, M P<0.001$ IC saline challenge vs IC cocaine challenge. ${ }^{+} P<0.05,{ }^{+}+P<0.01 \mathrm{BS}$ saline challenge vs BS cocaine challenge. ${ }^{\$} P<0.01,{ }^{\$ \$} P<0.001 \mathrm{BC}$ saline challenge vs $\mathrm{BC}$ cocaine challenge. ${ }^{\#} P<0.05 \mathrm{BS}$ cocaine challenge vs BC cocaine challenge; two-way ANOVA $(n=4-7)$. BC, binge cocaine; BS, binge saline; $c$, cocaine challenge; IC, intermittent cocaine; IS, intermittent saline; $\mathrm{s}$, saline challenge.

from the ventral tegmental area to the Nac occurs at the time of anticipation of reward. ${ }^{10,23-26}$

Strikingly, behavioral analysis indicated an increase in locomotor activity at the hours of cocaine injection in the IC group. This behavioral response may be in good agreement with the increase on DA release observed at the same hours, as it is well established that DA has a key role in motor function. Cocaine may induce a memory trace, which can be evaluated at behavioral and neurochemical levels during the anticipation phase of the drug administration. Interestingly, the 'neurochemical memory' was long lasting (WD1 and WD14) than the 'behavioral memory' (only WD1). This is the first demonstration of a neurochemical memory without cues associated to cocaine administration. This memory observed, by choosing the right time point for behavioral and neurochemical testing, can be due to a disturbance of the circadian rhythm. Indeed, it has been demonstrated that chronic exposure to drugs of abuse affects the circadian rhythm of physiological functions and behaviors. ${ }^{27-29}$ Moreover, psychostimulant-induced effects, such as behavioral sensitization and conditioned place preference, have been shown to follow circadian variations in their intensities. ${ }^{30-32}$

The second aim of our study was to examine the effects of acute cocaine challenge on locomotor activity and DA release. In animal models, a hallmark feature associated with chronic exposure to drugs of abuse, including cocaine, is locomotor sensitization. ${ }^{22,33,34}$ In some cases, this sensitized behavioral response is correlated with enhanced drug-induced DA responses in the $\mathrm{Nac}^{20,33-35}$ In this study, we demonstrated that the expression of a specific cocaine-induced behavior sensitization was related to the profile of administration. The binge treatment led to sensitization of locomotor effects of cocaine, whereas the intermittent treatment did not. These results are in good agreement with the literature, showing that patterns of administration are of particular importance, as they could determine duration and intensity of sensitization. 6,22,36 Interestingly, the locomotor sensitization observed in the binge group was associated to a DA release sensitization in the Nacc after a cocaine challenge on WD1. These results are supported by the reported increase in DA release in the Nacc in response to a subsequent challenge drug injection in animals previously exposed to cocaine. $22,33-35$

Regarding the neurochemical and behavioral consequences of the two patterns of cocaine administration important differences were observed. Interestingly, it clearly appeared from the experiments performed to evaluate the spontaneous behavior and basal extracellular levels of DA in the Nacc that the ability of the DA system to adapt to an event was maintained in the intermittent group, whereas it was impaired in the binge group. Indeed, exactly at the hours at which rats were habituated to receive a cocaine injection, a spontaneous increase in DA on WD1 until WD14, with an increase in locomotor activity on WD1, were observed in the former group, whereas only a spontaneous DA release on WD1 was measured in the latter group. Moreover, following acute cocaine challenge behavioral and neurochemical sensitizations were only observed in rats previously exposed to binge treatment. It is well known that chronic cocaine exposure induces enduring neuroadaptations that collectively result in the loss of control over drug-seeking behavior. In particular, locomotor sensitization, the augmented response to cocaine following repeated exposure, has been shown to 
have predictive validity for other indicators of addiction. Thus, in our experimental conditions, the binge pattern may be associated with the development of important behavioral and neurochemical modifications. In contrast, the intermittent pattern allowed rapid locomotor and DA increase when animals anticipated the drug administration, and did not lead to locomotor sensitization, suggesting that the addicted state was not completely installed and the system is still able to adapt to a specific event.

In conclusion, the results obtained clearly illustrate that the pattern of administration is a crucial parameter that should be taken into consideration while exploring the behavioral and neurochemical alterations in animals. Another very interesting result was that scheduling a cocaine injection on a daily basis led to expectation and anticipation in our experimental model. This circadian process could explain learned drug-taking patterns in which individuals search for drugs of abuse at specific hours of the day generally in a specific environment.

\section{Conflict of interest}

The authors declare no conflict of interest.

1. Mendelson JH, Mello NK. Management of cocaine abuse and dependence. N Engl J Med 1996; 334: 965-972.

2. Weiss F, Paulus MP, Lorang MT, Koob GF. Increases in extracellular dopamine in the nucleus accumbens by cocaine are inversely related to basal levels: effects of acute and repeated administration. J Neurosci 1992; 12: 4372-4380.

3. Unterwald EM, Kreek MJ, Cuntapay M. The frequency of cocaine administration impacts cocaine-induced receptor alterations. Brain Res 2001; 900: 103-109.

4. Schlussman SD, Zhou Y, Bailey A, Ho A, Kreek MJ. Steady-dose and escalating-dose "binge" administration of cocaine alter expression of behavioral stereotypy and striatal preprodynorphin mRNA levels in rats. Brain Res Bull 2005; 67: 169-175.

5. Bailey A, Gianotti R, Ho A, Kreek MJ. Downregulation of kappa-opioid receptors in basolateral amygdala and septum of rats withdrawn for 14 days from an escalating dose "binge" cocaine administration paradigm. Synapse 2007; 61: 820-826.

6. Le Marec T, Marie-Claire C, Noble F, Marie N. Chronic and intermittent morphine treatment differently regulates opioid and dopamine systems: a role in locomotor sensitization. Psychopharmacology (Berl) 2011; 216: 297-303.

7. Kalivas PW, Sorg BA, Hooks MS. The pharmacology and neural circuitry of sensitization to psychostimulants. Behav Pharmacol 1993; 4: 315-334.

8. NIH. Guide for the care and use of laboratory animals. Academic Press: Washington, DC, 1996.

9. Paxinos G, Watson C. The rat brain stereotaxic coordinates. 4th edn. Academic Press: San Diego, 1998.

10. Benturquia N, Le Marec T, Scherrmann JM, Noble F. Effects of nitrous oxide on dopamine release in the rat nucleus accumbens and expectation of reward. Neuroscience 2008; 155 341-344.

11. Rossetti ZL, Lai M, Hmaidan Y, Gessa GL. Depletion of mesolimbic dopamine during behavioral despair: partial reversal by chronic imipramine. Eur J Pharmacol 1993; 242 313-315.

12. Ventura R, Cabib S, Puglisi-Allegra S. Genetic susceptibility of mesocortical dopamine to stress determines liability to inhibition of mesoaccumbens dopamine and to behavioral 'despair' in a mouse model of depression. Neuroscience 2002; 115: 999-1007.

13. Maisonneuve IM, Ho A, Kreek MJ. Chronic administration of a cocaine "binge" alters basal extracellular levels in male rats: an in vivo microdialysis study. J Pharmacol Exp Ther 1995; 272: $652-657$.
14. Imperato A, Mele A, Scrocco MG, Puglisi-Allegra S. Chronic cocaine alters limbic extracellular dopamine. Neurochemical basis for addiction. Eur J Pharmacol 1992; 212: 299-300.

15. Rossetti ZL, Melis F, Carboni S, Gessa GL. Dramatic depletion of mesolimbic extracellular dopamine after withdrawal from morphine, alcohol or cocaine: a common neurochemical substrate for drug dependence. Ann N Y Acad Sci 1992; 654: 513-516.

16. Parsons $L H$, Smith AD, Justice JB Jr. Basal extracellular dopamine is decreased in the rat nucleus accumbens during abstinence from chronic cocaine. Synapse 1991; 9 : 60-65.

17. Robertson MW, Leslie CA, Bennett JP Jr. Apparent synaptic dopamine deficiency induced by withdrawal from chronic cocaine treatment. Brain Res 1991; 538: 337-339.

18. Zhang Y, Schlussman SD, Ho A, Kreek MJ. Effect of chronic 'binge cocaine' on basal levels and cocaine-induced increases of dopamine in the caudate putamen and nucleus accumbens of C57BL/6J and 129/J mice. Synapse 2003; 50: 191-199.

19. Segal DS, Kuczenski R. Repeated cocaine administration induces behavioral sensitization and corresponding decreased extracellular dopamine responses in caudate and accumbens. Brain Res 1992; 577: 351-355.

20. Kalivas PW, Duffy P. Time course of extracellular dopamine and behavioral sensitization to cocaine. I. Dopamine axon terminals. J Neurosci 1993; 13: 266-275.

21. Heidbreder CA, Thompson AC, Shippenberg TS. Role of extracellular dopamine in the initiation and long-term expression of behavioral sensitization to cocaine. J Pharmacol Exp Ther 1996; 278: 490-502.

22. Kalivas PW, Duffy P. Time course of extracellular dopamine and behavioral sensitization to cocaine. II. Dopamine perikarya. J Neurosci 1993; 13: 276-284.

23. Schultz W. Responses of midbrain dopamine neurons to behavioral trigger stimuli in the monkey. J Neurophysiol 1986; 56: 1439-1461.

24. Phillips PE, Stuber GD, Heien ML, Wightman RM, Carelli RM. Subsecond dopamine release promotes cocaine seeking. Nature 2003; 422: 614-618.

25. Wong DF, Kuwabara H, Schretlen DJ, Bonson KR, Zhou Y, Nandi A et al. Increased occupancy of dopamine receptors in human striatum during cue-elicited cocaine craving. Neuropsychopharmacology 2006; 31: 2716-2727.

26. Volkow ND. Addiction reviews. Introduction. Ann N Y Acad Sci 2008; 1141: xi-xii.

27. Gawin FH. Cocaine addiction: psychology and neurophysiology. Science 1991; 251: $1580-1586$

28. Vescovi PP, Coiro V, Volpi R, Passeri M. Diurnal variations in plasma ACTH, cortisol and beta-endorphin levels in cocaine addicts. Horm Res 1992; 37: 221-224.

29. Schierenbeck T, Riemann D, Berger M, Hornyak M. Effect of illicit recreational drugs upon sleep: cocaine, ecstasy and marijuana. Sleep Med Rev 2008; 12: 381-389.

30. Abarca C, Albrecht U, Spanagel R. Cocaine sensitization and reward are under the influence of circadian genes and rhythm. Proc Natl Acad Sci USA 2002; 99: 9026-9030.

31. Akhisaroglu M, Ahmed R, Kurtuncu M, Manev H, Uz T. Diurnal rhythms in cocaine sensitization and in Period1 levels are common across rodent species. Pharmacol Biochem Behav 2004; 79: 37-42.

32. Webb IC, Baltazar RM, Wang X, Pitchers KK, Coolen LM, Lehman MN. Diurnal variations in natural and drug reward, mesolimbic tyrosine hydroxylase, and clock gene expression in the male rat. J Biol Rhythms 2009; 24: 465-476.

33. Akimoto K, Hamamura T, Otsuki S. Subchronic cocaine treatment enhances cocaineinduced dopamine efflux, studied by in vivo intracerebral dialysis. Brain Res 1989; 490: 339-344.

34. Kalivas PW, Duffy P. Effect of acute and daily cocaine treatment on extracellular dopamine in the nucleus accumbens. Synapse 1990; 5: 48-58.

35. Pierce RC, Duffy P, Kalivas PW. Sensitization to cocaine and dopamine autoreceptor subsensitivity in the nucleus accumbens. Synapse 1995; 20: 33-36.

36. Vanderschuren LJ, Tjon GH, Nestby P, Mulder AH, Schoffelmeer AN, De Vries TJ. Morphine-induced long-term sensitization to the locomotor effects of morphine and amphetamine depends on the temporal pattern of the pretreatment regimen. Psychopharmacology (Berl) 1997; 131: 115-122

Translational Psychiatry is an open-access journal published by Nature Publishing Group. This work is licensed under the Creative Commons Attribution-NonCommercial-No Derivative Works 3.0 Unported License. To view a copy of this license, visit http://creativecommons.org/licenses/by-nc-nd/3.0/ 